\title{
Die Kernfamilie als Ideal. Zur Konstruktion von Scheidung und Nachscheidungsfamilien ${ }^{1}$
}

\author{
The nuclear family as an ideal. A view on constructing divorce and post- \\ divorce families
}

\section{Zusammenfassung:}

Vor dem Hintergrund hoher Scheidungsraten und veränderter sozialwissenschaftlicher Konzepte wird die Frage gestellt, wie Kinder und Eltern Scheidungen sowie familiale Lebensformen vor und nach einer Scheidung konstruieren. Basierend auf einer österreichischen qualitativen Befragung von 50 zehnjährigen Kindern und ihren Eltern (n = 71) zeigt sich, dass Scheidung überwiegend als Auflösung der Familie konstruiert wird. Kernfamilien werden, basierend auf den Aspekten Normalität, Komplementarität und Stabilität, als vorteilhafteste Lebensform wahrgenommen. EinEltern-Familien werden als defizitär und benachteiligt konstruiert, während Stieffamilien aufgrund der alltäglichen Präsenz von zwei Elternpersonen positiver betrachtet werden. Die Ergebnisse verweisen auf eine Orientierung am Desorganisationsmodell, eine Hierarchisierung von Lebensformen sowie haushaltszentrierte und familienstrukturell fokussierte Konzeptionen. Auswirkungen und Implikationen dieser Ergebnisse für Sozialpolitik und Familienforschung werden diskutiert.

\begin{abstract}
:
Against the backdrop of high divorce rates and changing concepts in the social sciences, the issue is addressed as to how children and families construct divorce and patterns of family life prior and subsequent to divorce. Based on an Austrian qualitative survey of 50 ten-year old children and their respective parents $(n=71)$, it can be shown that divorce is predominantly constructed as a disintegration of the family. Based upon the dimensions of normalcy, complementarity and stability, nuclear families are being perceived as the most advantageous form of living together as a family. Whereas single-parent families are constructed as being both deficient and disadvantaged, stepfamilies are seen in a more positive light which, in turn, is due to the everyday presence of two parents in those families. These findings indicate that the interviewees orientate themselves along the lines of the model of disorganization, hierarchized ways of family life, and family concepts that are household-centered and focus primarily on family structures. Finally, the implications and repercussions of these findings for family policies and family research are being discussed.
\end{abstract}

1 Für wertvolle Kommentare danke ich den anonymen Gutachter(inne)n. Mein herzlicher Dank für die Diskussion früherer Versionen gilt Lieselotte Wilk, Cornelia Schadler, Barbara Haas sowie Elisabeth Scheibelhofer. 
Schlagworte: Scheidung, Kinder geschiedener Eltern, Ein-Eltern-Familie, Stieffamilie, Kernfamilie, qualitative Interviews
Key words: divorce, children of divorce, singleparent families, stepfamilies, nuclear families, qualitative interviews

\section{Einleitung}

Im Verlauf der letzten Jahrzehnte sind die Scheidungszahlen in nahezu allen europäischen Ländern angestiegen. Im Zuge dieser Entwicklung ist auch eine steigende normative Akzeptanz von Scheidung zu beobachten, die sich sowohl in Einstellungsumfragen (Wernhart/Neuwirth 2007; Hamachers-Zuba et al. 2009) als auch in einem veränderten sozialwissenschaftlichen Verständnis abbildet (Théry 1988; Herzer 1998; Hill/Kopp 1999): die Betrachtung von Scheidung als dysfunktionales, die Familie auflösendes Ereignis im Rahmen eines Desorganisationsmodells wurde durch Reorganisationsmodelle abgelöst, welche Scheidung als normativ akzeptierten Prozess in der Familienentwicklung betrachten. Diese Entwicklungen deuten darauf hin, dass wir eine ,neue Ära von Scheidungskonzeptionen“ (Adams/Coltrane 2007: 17) betreten haben: von der Konstruktion von Scheidung als defizitärem Ereignis hin zu Scheidung als gesellschaftlich akzeptiertem Phänomen, das kaum normative Benachteiligungen mit sich bringt. Gleichzeitig verweisen Forschungsarbeiten aus dem Bereich der Familienrhetorik (Lüscher 1997; Cyprian 2003) auf die weiterhin bestehende Bedeutung der Kernfamilie als hegemoniales Familienleitbild.

Wie werden nun angesichts einer gestiegenen normativen Akzeptanz von Scheidungen familiale Leitbilder und Vorstellungen über familiale Lebensformen konstruiert? Basierend auf qualitativen Interviews mit zehnjährigen Kindern und deren Eltern (Zartler et al. 2009) wird die Frage gestellt, wie diese Scheidung, Kernfamilien und Nachscheidungsfamilien (Stieffamilien, Ein-Eltern-Familien ${ }^{2}$ ) konstruieren. Zugrunde liegt eine sozialkonstruktivistische Herangehensweise, welche die subjektiven Deutungsmuster in den Vordergrund rückt (Berger/Kellner 1965; Berger/Luckmann 1969). Der Beitrag bezieht sich auf Scheidungen von Paaren mit minderjährigen Kindern sowie auf die Zuschreibungen der Befragten an die Fähigkeiten und Möglichkeiten unterschiedlicher familialer Lebensformen, eine förderliche Umgebung für Kinder bereitzustellen. Die Ergebnisse bisher vorliegender Arbeiten zur Konstruktion und Konfiguration von Scheidung und Nachscheidungsfamilien (Schmitz/Schmidt-Denter 1999; Smart et al. 2001; Roe et al. 2006; Castrén 2008, Zartler 2011) werden in dieser Analyse erweitert.

2 Stieffamilien und Ein-Eltern-Familien können auch andere Entstehungsursachen haben; häufig stehen sie jedoch in Zusammenhang mit einer Scheidung bzw. Trennung. Aufgrund ihrer Etablierung im wissenschaftlichen und öffentlichen Diskurs werden die Begrifflichkeiten Stieffamilie, EinEltern-Familie bzw. Alleinerziehende verwendet. Es wird darauf hingewiesen, dass diese bereits negative Konnotationen bzw. defizitäre Zuschreibungen implizieren. 


\section{Sichtweisen über Scheidung: empirische Befunde und theoretische Konzeptionen}

Europäische Länder weisen heute deutlich höhere Scheidungsraten auf als vor einigen Jahrzehnten (OECD 2010). Dies gilt auch für Österreich: Betrug die Gesamtscheidungsrate $^{3}$ im Jahr 1961 noch 14\% (Statistik Austria 2011c), so liegt sie im Jahr 2010 bei 43\%. Knapp zwei Drittel (62\%) der Scheidungen des Jahres 2010 betrafen Ehen mit Kindern, rund 14.000 minderjährige Kinder erlebten in diesem Jahr die Scheidung ihrer Eltern (Statistik Austria 2011a). Das „Scheidungsrisiko“ aus Sicht der Kinder, d.h. die Wahrscheinlichkeit, vor dem 18. Geburtstag eine Scheidung der Eltern zu erleben, betrug $20 \%{ }^{4}$. Die häufigste Familienform, in der Kinder nach einer elterlichen Trennung oder Scheidung leben, ist die mütterliche Ein-Eltern-Familie. 13\% aller österreichischen Kinder unter 15 Jahren lebten im Jahr 2010 in einer Ein-Eltern-Familie, davon 94\% mit einer alleinerziehenden Mutter und 6\% mit einem alleinerziehenden Vater ${ }^{5}$ (Statistik Austria 2011b, 70). Insgesamt waren 14,6\% aller Familien mit Kindern unter 15 Jahren EinEltern-Familien (Statistik Austria 2011b: 70). Stieffamilien machten 9,7\% aller Paarfamilien mit Kindern unter 15 Jahren aus; jedes zehnte Kind dieser Altersgruppe lebte in einer Stieffamilie (Statistik Austria 2011b: 80, 85).

Einstellungsumfragen verweisen auf eine europaweit steigende gesellschaftliche Akzeptanz von Scheidungen, wobei Scheidungen kinderloser Ehen breitere Akzeptanz finden als solche in Familien mit Kindern (Halman et al. 2005; Diefenbach/Opp 2007; Kalmijn/Uunk 2007; Toth/Kemmelmeier 2009). In Österreich stimmen 83\% der im Rahmen des ISSP (International Social Survey Programme) Befragten der Aussage zu, dass eine Scheidung im Allgemeinen die beste Möglichkeit zum Umgang mit unlösbaren Eheproblemen darstellt (Wernhart/Neuwirth 2007). Auch die europäische Wertestudie zeigt zunehmend permissivere Haltungen gegenüber Scheidung: Während im Jahr 1990 ein Drittel (32\%) eine Scheidung keinesfalls in Ordnung fand, galt dies 2008 für ein Fünftel (22\%) (Hamachers-Zuba et al. 2009).

In der sozialwissenschaftlichen Forschung ist eine grundlegende Veränderung theoretischer Perspektiven festzustellen. Scheidung wurde bis weit ins 20. Jahrhundert im Rahmen eines Defizit- oder Desorganisationsmodells als moralische Verfehlung gesehen, welche die Auflösung familialer Beziehungen, die Übertragung des Sorgerechts auf einen Elternteil und eine untergeordnete Rolle des anderen implizierte (Théry 1988; Herzer 1998; Hill/Kopp 1999). Seit den 1980er Jahren wird Scheidung im Rahmen eines Transitions- oder Reorganisationsmodells als normativ akzeptierte Veränderung betrachtet, welche eine Neuorganisation des familialen Beziehungssystems bedeutet und weiterhin beide Elternteile inkludiert (Cowan/Hetherington 1991; Fthenakis 1995; Amato 2000). Entspre-

3 Die Gesamtscheidungsrate gibt an, wie viele der im Berichtsjahr geschlossenen Ehen bei gleichbleibendem Scheidungsverhalten voraussichtlich künftig geschieden werden.

4 Auskunft von Anita Mikulasek, Statistik Austria, 20.6.2011.

5 Vier von zehn dieser Kinder hatten geschiedene Eltern, in ebenso vielen Fällen war der alleinerziehende Elternteil ledig, wobei vermutlich zum Teil eine Trennung vorausgegangen war. Weitere 15,6\% der unter 15-Jährigen in Ein-Eltern-Familien hatten einen verheirateten, aber getrennt lebenden Elternteil; in 3,5\% der Fälle war der alleinerziehende Elternteil verwitwet (Statistik Austria 2011b: 81). 
chend haben sich auch die Sichtweisen, wie Kinder geschiedener Eltern sozialwissenschaftlich betrachtet werden, verändert: Sie werden nicht länger als passive Opfer einer elterlichen Scheidung gesehen, sondern als aktive, eigenständige Subjekte, die ihr Leben kompetent mitgestalten können (Smart et al. 2001; Flowerdew/Neale 2003; Robinson et al. 2003; Smith et al. 2003).

Trotz dieser Entwicklungen auf der Einstellungsebene und im sozialwissenschaftlichen Diskurs werden auch normierend-moralisierende Tendenzen sichtbar (Coltrane/Adams 2003). Familienrhetorische Arbeiten (Lüscher et al. 1989; Lüscher 1997; Cyprian 2003) verweisen darauf, dass Familiendefinitionen oftmals ehezentriert, heteronormativ bzw. auf die Eltern-Kind-Beziehung fokussiert sind (Oswald et al. 2005; Nave-Herz 2006; Schneider 2008; Lange/Kränzl-Nagl 2010). Die Kernfamilie, bestehend aus Vater, Mutter und gemeinsamen Kindern, fungiert weiterhin als „,ideologischer Code“ (Smith 1993) und als Vergleichsfolie in normativer, kultureller, rechtlicher und politischer Hinsicht. Sie zeichnet sich als hegemoniales Familienleitbild ab (Toppe 2009) und wird für das Aufwachsen von Kindern vielfach als beste Umgebung betrachtet, auch wenn differenzierte Forschungsbefunde dem entgegen stehen (Röhr-Sendlmeier/Greubel 2004; Wendt/Walper 2007; Schneewind/Walper 2008; Alt/Lange 2001). Darüber hinaus gibt es zahlreiche Hinweise auf kritische Haltungen gegenüber Nachscheidungsfamilien (Miles/Servaty-Seib 2010; Moon 2011), wie z.B. eine Benachteiligung von Kindern geschiedener Eltern im Urteil ihrer Lehrer(innen) (Sander et al. 2005), eine negative Charakterisierung von EinEltern-Familien (Moxnes 2003; Usdansky 2009) und der Ausrichtung ihres Familienlebens an kernfamilialen Strukturen (Nelson 2006). Aus Kindersicht zeigt sich, dass bestimmte nicht-kernfamiliale Lebensformen, wie Stieffamilien oder alleinerziehende Mütter mit einem nicht im Haushalt lebenden Partner, von Kindern vielfach nicht als Familien wahrgenommen werden (Rigg/Pryor 2007) und dass die Familiendefinitionen von Kindern - weitgehend unabhängig von der Lebensform, in der sie selbst aufwachsen - oftmals kernfamiliale Strukturen widerspiegeln (Watson/Amgott-Kwan 1984; Anyan/Pryor 2002; Alt/Lange 2004).

\section{Datenbasis}

Die empirische Grundlage dieses Beitrags ist eine qualitative Erhebung mit 50 zehnjährigen Kindern (20 Jungen, 30 Mädchen) und ihren Eltern (71 Eltern, davon 25 Väter und 46 Mütter) (Zartler et al. 2009). Die Befragung von Kindern und ihren Eltern ermöglicht die Erfassung multipler Perspektiven innerhalb einer Familie (Ribbens McCarthy et al. 2003; Dekovic/Buist 2005; Harden et al. 2010; Zartler 2010). Zu berücksichtigen ist aufgrund des Alters der befragten Kinder, dass soziale Werte und Normen zunächst vorwiegend in der Familie erlernt werden (Hurrelmann 2002; Geulen 2010) und diese das kindliche „,Weltbild über das Sein in Familie“ beeinflusst (Hölscher 2008, 758, Hervorhebung im Original). Zehnjährige Kinder befinden sich aus entwicklungstheoretischer Sicht (Piaget 1972; Kohlberg 1974; Oerter/Montada 2008; Miller 2011) kognitiv am Übergang zur Phase der formalen Operationen, wo abstraktes Denken möglich wird. Was die Übernahme von Normen betrifft, so richten jüngere Kinder sich zunächst stark nach den elterlichen Vorgaben und beginnen in Abwägung mehrerer und unterschiedlicher As- 
pekte und unter Berücksichtigung übergreifender ethischer Werte selbständige moralische Urteile zu entwickeln (Piaget 1986; Birney/Sternberg 2011; Denham et al. 2011).

Für die Befragung der Kinder wurden Foto-Interviews verwendet (Clark-Ibáñez 2004; Capello 2005; Cook/Hess 2007; Croghan et al. 2008): Die Kinder erstellten zunächst mittels Einwegkameras Fotos ihrer eigenen Lebenswelt in fünf vorgegebenen Bereichen ${ }^{6}$. Im Interview wurden sie dann gebeten, die ausgearbeiteten Fotos zu kommentieren und über die abgebildeten Personen und Lebensbereiche zu erzählen. Zusätzlich wurde ein Interview-Leitfaden ${ }^{7}$ als Strukturierungshilfe verwendet. Mit den Eltern (jeweils mindestens ein Elternteil) wurden problemzentrierte Leitfadeninterviews (Witzel 2000) geführt und im Anschluss an das Interview soziodemografische Daten erhoben. Die von den Kindern erstellten Fotos wurden in den Interviews mit ihren Eltern nicht verwendet. Alle Interviews wurden als Einzelgespräche geführt und vollständig transkribiert.

Die Datenerhebung erfolgte in der Großstadt Wien (1,7 Mio. Einwohner(innen)), die zugleich Österreichs wichtigster Wirtschaftsstandort ist, und in einem Ort mit 5.000 Einwohner(inne)n im Burgenland, Österreichs östlichstem Bundesland, welches ein wirtschaftlich und infrastrukturell eher benachteiligtes Gebiet repräsentiert. Scheidungen, EinEltern-Familien und Stieffamilien sind im städtischen Erhebungsgebiet häufiger als im ländlichen ${ }^{8}$. Die einbezogenen Familien sind in beiden Erhebungsgebieten hinsichtlich soziodemografischer Faktoren wie Erwerbs- oder Bildungsstatus der Eltern heterogen. Es sind unterschiedliche Lebensformen vertreten, wobei insbesondere darauf Bedacht genommen wird, ob die befragten Kinder Veränderungen der Familienstruktur erlebt haben $^{9}$, in einer nicht-kernfamilialen Lebensform oder in einer Kernfamilie aufwachsen. Auf Basis der Kriterien Rollenbesetzung, Familienbildungsprozess und Wohnsitz werden in der vorliegenden Studie Kernfamilien definiert als Familien, in welchen ein (verheiratetes oder unverheiratetes) Elternpaar mit dem/den gemeinsamen Kind(ern) im Haushalt lebt und es keine weiteren inner- oder außerhalb dieser Familie lebenden Kinder der Eltern bzw. eines Elternteils gibt. Die gemeinsamen Kinder wachsen seit ihrer Geburt mit dem Elternpaar auf und haben keine elterliche Trennung bzw. Scheidung ${ }^{10}$ erlebt. Diesen Kriterien entsprechen 28 Familien (neun aus dem städtischen, 19 aus dem ländlichen Erhebungsgebiet). Als nicht-kernfamilial gelten jene Lebensformen, welche zumindest eines

6 Fotos wurden zu folgenden Bereichen erstellt: (1) Meine Familie stellt sich vor. Wer gehört zu meiner Familie?; (2) Was mir besonders gut in meiner Familie gefällt; (3) Was mir weniger in meiner Familie gefällt; (4) Was wir in unserer Freizeit machen (unter der Woche); (5) Was wir am Wochenende machen.

7 Die Interview-Leitfäden für Kinder und für Eltern deckten die Bereiche Familienformen und Scheidung, Familienzeit sowie kindliche Partizipation in der Familie ab.

8 Die Scheidungsrate ist in Wien bundesweit am höchsten und lag im Erhebungsjahr 2009 bei 54\% (Burgenland: 43\%) (Statistik Austria 2010a). Der Anteil an Ein-Eltern-Familien war in Wien mit 14\% bundesweit am höchsten (Burgenland: 9\%), ebenso jener an Stieffamilien (11\% in Wien, 9\% im Burgenland) (Statistik Austria 2010b: 72, 76) .

9 Es werden nur bereits vom Kind erlebte familienstrukturelle Veränderungen inkludiert. Von den Eltern bzw. einem Elternteil erwähnte, künftig erwartete Veränderungen wie eine geplante Trennung wurden nicht berücksichtigt. Im Falle uneinheitlicher Darstellungen der einzelnen Familienmitglieder orientieren wir uns an den Aussagen des Kindes.

10 Im Folgenden werden die Begriffe Scheidung und Trennung synonym verwendet und darunter eine räumliche Trennung in Kombination mit einer Beendigung der elterlichen Partnerbeziehung verstanden. 
dieser Kriterien nicht erfüllen. Dies trifft auf 22 Familien zu (zehn aus dem städtischen, zwölf aus dem ländlichen Erhebungsgebiet ${ }^{11}$. Zehn Kinder aus diesen Familien erlebten eine Trennung der Eltern (je fünf in jedem Erhebungsgebiet); zwei burgenländische Kinder erlebten, jeweils im Alter von sechs Jahren, den Tod eines Elternteils. Zehn Kinder aus nicht-kernfamilialen Lebensformen erlebten keine elterliche Trennung, da diese bereits vor ihrer Geburt stattfand oder sie als leibliches Kind in einer Stieffamilie leben.

Die Datenauswertung erfolgte mit einer Kombination qualitativer Methoden, wobei zum Teil in Gruppen gearbeitet wurde, um dem Anspruch nach intersubjektiver Nachvollziehbarkeit entsprechen zu können. Im ersten Schritt wurden angelehnt an das Codierverfahren der Grounded Theory ausgewählte Interviewtranskripte offen codiert und ein induktives Kategoriensystem entwickelt (Strauss/Corbin 1990; Glaser/Strauss 1998). In einem zweiten Schritt wurde auf dieser Basis eine qualitative Themenanalyse (Froschauer/Lueger 2003) als interpretativ-reduktives Verfahren durchgeführt ${ }^{12}$. Damit konnten charakteristische Elemente sowie Unterschiede in der Themendarstellung herausgearbeitet, Kernaussagen eruiert und der Kontext ihres Auftretens ermittelt werden. Einige Kategorien werden in der Ergebnisdarstellung wörtlich unter einfachen Anführungszeichen wiedergegeben. Zur Illustration werden charakteristische Passagen wörtlich zitiert ${ }^{13}$.

\section{Ergebnisse}

\subsection{Scheidung}

\section{Scheidung als verbreitetes, gesellschaftlich akzeptiertes Phänomen}

Scheidung wurde von den befragten Eltern als verbreitetes Phänomen beschrieben und das Ansteigen der Scheidungszahlen als eine nicht aufzuhaltende gesellschaftliche Entwicklung wahrgenommen. Dies resultierte auch aus einer quantitativen Überschätzung: der Anteil geschiedener Personen wurde mit ,jede/r Zweite“ bis ,jede/r Vierte“ geschätzt. Aus dieser (vermuteten) quantitativen Verbreitung von Scheidungen leiteten die befragten Eltern eine erhöhte gesellschaftliche Akzeptanz und Toleranz ab. Scheidung stelle heute ,"nichts Besonderes mehr" dar (Vater B $03^{14}$, Kernfamilie), weshalb auf gesellschaftlicher Ebene ein hohes $\mathrm{Ma} ß$ an Offenheit entstanden sei. Negative Sanktionen seien demnach seltener als früher. Regionale Unterschiede bestehen dahingehend, dass die befragten Eltern aus dem städtischen Erhebungsgebiet eine deutlich geringere Toleranz gegenüber

11 Neun der befragten Kinder lebten zum Befragungszeitpunkt in einer Ein-Eltern-Familie (vier in Wien, fünf im Burgenland). Elf Kinder lebten in einer Stieffamilie (sechs in Wien, fünf im Burgenland), zwei burgenländische Kinder in einer Pflegefamilie.

12 Angesichts des multiperspektivischen Vorgehens ergeben sich auch andere Auswertungsmöglichkeiten als sie im Rahmen dieses Beitrags dargestellt werden können. So ist zu erwarten, dass eine Auswertung auf Familienebene weitere spezifische Aufschlüsse geben könnte.

13 Wörtliche Zitate sind kursiv gedruckt und in doppelte Anführungszeichen gesetzt.

14 B steht für Burgenland, W für Wien. In Hinblick auf bessere Lesbarkeit wurden Dialektausdrücke in Schriftdeutsch übertragen. Alle Namen sind anonymisiert. Längere Auslassungen sind mit [...] gekennzeichnet. 
Scheidung und daraus resultierenden nicht-kernfamilialen Lebensformen in ländlichen Regionen vermuteten. Die befragten Eltern aus dem ländlichen Erhebungsgebiet konstatierten hingegen eine zunehmende Angleichung städtischer und ländlicher Regionen.

Die befragten Kinder setzten eine Scheidung mit der Entstehung neuer Familienstrukturen gleich und schätzten die Zahlen deutlich niedriger ein als ihre Eltern. Exemplarisch sagte ein Mädchen über die familialen Strukturen im Klassenverband: „Ein Dreiviertel hat Ganz-Familien und ein Viertel hat eben getrennte Familien. Und da gehöre ich auch dazu. " (Mädchen W 10, Stieffamilie)

\section{Scheidung als ,Auflösung der Familie‘}

Trotz der wahrgenommenen Toleranz auf gesellschaftlicher Ebene wurde Scheidung bzw. Trennung in Familien mit Kindern von den Interviewpartner(innen) äußerst kritisch und negativ beurteilt. Dabei fanden sich nur wenige Unterschiede in den Einschätzungen der Befragten (Kinder und Eltern) mit und ohne Trennungserfahrung, ebenso wenig zwischen den beiden Erhebungsgebieten. Scheidung führe, so der Haupttenor, zwingend zu Diskontinuität und Belastungen. Die in den Interviews präsentierten Sichtweisen beinhalteten zentrale Aspekte des Defizitmodells.

Die befragten Kinder belegten eine elterliche Scheidung mit Attributen wie ,mühsam “, , ,dumm “, „nicht toll“, ,traurig“, ,,urblöd" oder ,nicht so cool". Scheidung wurde unabhängig davon, in welcher Lebensform die Kinder aufwuchsen und ob sie eine elterliche Scheidung erlebt hatten - erstens gleichgesetzt mit dem Verlust des Vaters bzw. gravierenden Veränderungen in der Beziehung zum Vater. Dies wurde zweitens als emotional belastend und als Einschränkung der kindlichen Handlungsmöglichkeiten interpretiert. Beide Aspekte werden im Folgenden illustriert:

„Eine Scheidung ist nicht so gut. Weil dann hätte man keinen Papa, und das wär natürlich dumm, weil man dann nicht so viel machen könnte. Weil jetzt bei uns, wenn die Mama keine Zeit hat, kann ich mit'm Papa was machen. Und wenn der Papa keine Zeit hat und die Mama Zeit hat, kann ich mit der Mama was machen. "(Junge B 17, Stieffamilie).

Der Vergleich zwischen Kindern mit und ohne Trennungserfahrung zeigt: Die befragten Kinder mit geschiedenen Eltern betonten ebenfalls negative Aspekte, reflektierten aber auch über moderierende Faktoren wie den Umgang der beteiligten Erwachsenen mit der neuen Familiensituation, die Gestaltung von Besuchs- und Obsorgeregelungen sowie Kommunikation und Information über die (geplante) Scheidung. Unabhängig davon, ob die Kinder selbst eine elterliche Scheidung erlebt hatten, wurden auch positive Assoziationen artikuliert, wie z.B. eine mögliche Reduktion elterlicher Konflikte, materielle Vorteile wie das Vorhandensein von Spielsachen in beiden elterlichen Haushalten sowie ein vergrößerter Gestaltungsspielraum: ,"In einer gerissenen Familie hat man zwei Welten, und man kann sie immer wechseln. " (Mädchen W 10, Stieffamilie). Der Umgang mit Prozessen der Familientransition im Freundeskreis bzw. Klassenverband wurde als problematisch und verunsichernd beschrieben. Kommunikation darüber gäbe es höchst selten - u.a. aufgrund der Sorge, durch solche Gespräche Emotionen wie Traurigkeit oder Wut bei den Freund(inn)en auszulösen und damit nicht adäquat umgehen zu können.

Die befragten Eltern beurteilten eine Scheidung ebenfalls überwiegend als negatives, Stress verursachendes Ereignis, welches umfassende Anforderungen an alle Familien- 
mitglieder stellen und „unvollständige“, ,zerrissene“ Familien zurücklassen würde auch wenn konstatiert wurde, dass eine Scheidung in manchen Fällen eine positive Möglichkeit zur Beendigung einer konflikthaften, belastenden oder unbefriedigenden Partnerbeziehung sei. Dabei wurden - unabhängig von eigenen Scheidungserfahrungen - Unterschiede nach der aktuellen Lebensform der Befragten deutlich: Befragte in Zwei-ElternFamilien (Kern- und Stieffamilien) wiesen kritischere Haltungen gegenüber einer Scheidung auf als jene in Ein-Eltern-Familien. Eltern in Kernfamilien zeigten sich am kritischsten. Sie konstruierten Scheidung als schmerzhaften Prozess, der Kindern Verletzungen zufüge, wörtlich als ,eine Wunde, die heilen muss “ (Mutter W 15, Kernfamilie). Die durch eine Scheidung entstehenden Defizite seien für Kinder nicht ausgleichbar:

„Es fehlt irgendwo in der Seele ein Stück. Es fehlt immer ein Teil, und das ist der Unterschied, glaub ich. [...] Irgendwo fehlt was. Wenn ein Mann fehlt, fehlt diese Seite, wenn die Mama fehlt, dann fehlt die andere Seite. " (Vater B 10, Kernfamilie).

Geschiedenen Eltern wurde zugeschrieben, dass ihr „Verantwortungsgefühl von vornherein ganz anders aufgebaut" sei (Mutter W 08, Kernfamilie) und vermutet, dass der Entschluss zur Trennung vielfach eine unreflektierte Entscheidung darstelle, die auch aus zu geringem elterlichen Bemühen resultiere. Die befragten Eltern aus Kernfamilien - und überwiegend auch jene aus Stieffamilien - distanzierten sich von geschiedenen Eltern und charakterisierten diese als (zu) wenig belastungsfähig und (zu) wenig bereit, für den Erhalt der Partnerbeziehung zu kämpfen.

Auch die befragten Eltern in nicht-kernfamilialen Lebensformen (Alleinerziehende sowie einige geschiedene Eltern in Stieffamilien) konstruierten Scheidung vorwiegend als negatives Ereignis mit gravierenden Auswirkungen für Kinder, maßen allerdings dem Umgang der beteiligten Erwachsenen mit der Trennungssituation besonders große Bedeutung zu. Sie nannten eine Reihe von moderierenden Variablen und betonten die zentrale Bedeutung von elterlicher Kooperation, Kommunikation und Rücksichtnahme auf kindliche Bedürfnisse. Zum Teil wurden von diesen Eltern auch positive Aspekte thematisiert: So könnten Kinder die Erfahrung machen, dass Beziehungsarbeit und gegenseitige Unterstützung notwendig sei, um eine Partnerbeziehung aufrecht $\mathrm{zu}$ erhalten, und sie könnten im Idealfall von einer verstärkten Involvierung ihrer Väter nach der Scheidung profitieren. Gleichzeitig wurde aber betont, dass beide Effekte ihrer Erfahrung nach nur selten einträfen.

\section{Kinder mit geschiedenen Eltern als bemitleidenswerte ,Scheidungswaisen}

Eine elterliche Scheidung wurde als ganz besonders nachteilig für die betroffenen Kinder betrachtet: Ihnen wurden Attribute wie „arm “, ,, verunsichert" oder „,verstört “ zugeschrieben und die kindliche Rolle bei einer elterlichen Scheidung als passiv und machtlos konstruiert: „Die werden hin und her geschubst und wissen nicht genau, wo sie hingehören. " (Mutter B 14, Kernfamilie). Jene Emotion, die am häufigsten gegenüber Kindern geschiedener Eltern thematisiert wurde, war Mitleid. Eine Scheidung wurde als stresshaftes Ereignis für Kinder konstruiert, welches zwingend zu Überforderung und Benachteiligung führen würde. Argumentiert wurde dies mit dem einer Scheidung gleichgesetzten ,Verlust' eines Elternteils (nämlich des Vaters) und der daraus resultierenden Betrachtung von Kindern als ,Scheidungswaisen'. Nach einer Scheidung sei nur noch ein Elternteil 
verfügbar, welcher dem Kind Sicherheit und Stabilität geben könne, und das Kind hätte „,keine einheitliche Heimat" mehr (Mutter W 09, Stieffamilie). Kinder geschiedener Eltern würden, so die Vorstellung, im Vergleich zu jenen aus Kernfamilien deutlich weniger Harmonie in der Familie erleben.

Diese negativen Sichtweisen wurden von Kindern und Eltern aus Kernfamilien sowie nicht-kernfamilialen Lebensformen präsentiert - und zwar unabhängig davon, ob sie selbst eine Scheidung erlebt hatten. Relativierungen wurden lediglich von einigen Eltern in nicht-kernfamilialen Lebensformen getroffen, welche die Ressourcen und Fähigkeiten der beteiligten Personen sowie die Relevanz der Rahmenbedingungen betonten.

\subsection{Die Kernfamilie als Ideal}

Kernfamilien, in welchen die Eltern mit ihren leiblichen Kindern in einem gemeinsamen Haushalt leben, wurden von den befragten Kindern und Eltern in Kern- und Stieffamilien als vorteilhaft für alle involvierten Personen, insbesondere Kinder, betrachtet. Dies basierte auf drei Argumentationslinien: Normalität, Komplementarität und Stabilität.

Normalität wurde Kernfamilien in quantitativer und qualitativer Hinsicht zugeschrieben. Dies zeigte sich an den verwendeten Begrifflichkeiten wie ,normal ", ,richtig “, „typisch“, „klassisch“ oder ,konventionell“ sowie an der Zuschreibung von Harmonie zu dieser Lebensform. Kernfamilien wurden als ,,intakt “, ,komplett", ,funktionierend" oder „optimal" bezeichnet. Familien, in welchen eine Scheidung stattgefunden hat, wurden umgekehrt als ,,zerrissene ", ,, unvollständige “ oder ,, unorthodoxe“" Familien etikettiert.

Zweitens wurde Komplementarität, resultierend aus dem Vorhandensein zweier Elternteile, als zentral erachtet. Die alltägliche Präsenz zweier Elternteile ermögliche, so die in den Interviews überwiegende Vorstellung, die Aufteilung von Erziehungsaufgaben, denn es sei ,immer jemand da, auf den man sich verlassen kann. “ (Mutter W 15, Kernfamilie). Die Befragten gingen davon aus, dass das Erleben einer Dualität von Geschlechterrollen in der Familie per se förderliche Bedingungen für die kindliche Entwicklung bieten würde ${ }^{15}$.

Mit ihrer Komplementarität sei, so die überwiegend zum Ausdruck gebrachte Vorstellung, die Stabilität der Kernfamilie verknüpft. Die Kernfamilie würde Kindern per se Schutz, Sicherheit und Geborgenheit geben. Sie sei eine „sichere Zelle, die eine wahnsinnige Stabilität in jeder Form " bieten würde (Mutter W 06, Kernfamilie). Kinder geschiedener Eltern würden, so die Vorstellung, diese Stabilität nicht erleben und sie verstärkt bei ihren Freund(inn)en in kernfamilialen Lebensformen suchen. So beschrieb ein Junge (B 22, Kernfamilie) seinen Vater als „Ersatzpapa” für seinen besten Freund, da diesem , halt der Papa fehlt.” Kernfamilien wurden als ,sicherer Hafen“ für Kinder, die eine elterliche Scheidung erlebt haben, betrachtet.

Die befragten Eltern aus Kernfamilien schrieben ihrer Lebensform eine gewisse moralische Überlegenheit zu: Sie wären bereit, Konflikte konstruktiv zu bearbeiten und würden nicht leichtfertig und vorschnell ihre Partnerbeziehung aufgeben. Dies sei einerseits auf eigenes Bemühen und individuelle Stärken (wie Disziplin, Pflichtbewusstsein und

15 Familien, in denen gleichgeschlechtliche Eltern mit ihren Kindern leben, wurden in keinem der vorliegenden Interviews erwähnt. 
Durchhaltevermögen) zurückzuführen, basiere andererseits aber auch auf einer glücklichen Fügung: „Ich glaube, dass alle Kreuze schlagen, dass sie eben in stabilen Familienverhältnissen leben, dass ihnen das vergönnt ist. "(Mutter W 06, Kernfamilie).

Zusammenfassend wurde von den Befragten in Zwei-Eltern-Familien die Kernfamilie mit einer Fülle an positiven Aspekten konnotiert. Zwei Befragtengruppen äußerten allerdings auch kritische bzw. negative Aspekte: Alleinerziehende sowie Mütter in Kernfamilien, die sich aufgrund eines hohen Erwerbs- und geringen familialen Engagements ihres Partners als temporäre Alleinerzieherinnen fühlten. Von diesen beiden Gruppen wurden Kernfamilien mit veralteten Werten und Idealbildern in Verbindung gebracht und eine Diskrepanz zwischen innerfamiliären Konflikten und nach außen demonstrierter Harmonie vermutet (,Doppelmoral'). Ebenso wurde hinterfragt, ob das bloße Vorhandensein zweier Elternteile eine gerechte Verteilung von Verantwortlichkeiten garantieren könne. Es wurde darauf verwiesen, dass eine permanente Verfügbarkeit beider Elternteile in Kernfamilien allein aufgrund beruflicher Notwendigkeiten und zeitlicher Restriktionen kaum möglich sei. Gleichzeitig wurde angemerkt, dass manche Väter sich auch nach einer Scheidung umfassend für ihre Kinder engagieren würden, und betont, dass eine dichotome Sichtweise zu kurz greife und eine Idealisierung der Kernfamilie nicht gerechtfertigt erscheine.

\subsection{Nachscheidungsfamilien}

\section{Ein-Eltern-Familien als , Mangelfamilien ‘}

Ein-Eltern-Familien wurden in der vorliegenden Befragung als defizitäre und besonders herausfordernde Lebensform betrachtet. Sie wurden von den befragten Kindern aus Kernfamilien und Stieffamilien mit Attributen wie ,,schwieriger “, ,, anstrengender " und ,komplizierter" versehen. Als größtes Defizit wurde das ,Fehlen" eines Elternteils betrachtet:

„Nur mit einem Elternteil leben, das ist nicht so besonders, weil jedes Kind braucht zwei Elternteile.“ - ,Warum?" - „Also, ähm, damit es sich besser fühlt, also dass man sich in einer richtigen Familie fühlt, dass da alle zusammen sind, Mama und Papa. " (Mädchen W 15, Kernfamilie)

Die Abwesenheit eines zweiten Elternteils wurde sowohl im Familienalltag als auch in Bezug auf Freizeitaktivitäten, Feste und Schulveranstaltungen als gravierender Nachteil betrachtet. Die befragten Kinder aus Stieffamilien grenzten sich von den Kindern alleinerziehender Eltern ab, da hier lediglich ein Elternteil im Alltag präsent sei. Dies führe, so die Vermutung, dazu, dass Kinder alleinerziehender Eltern nur wenig Zeit mit ihrem Elternteil verbringen könnten und dadurch benachteiligt seien, wie ein Mädchen über seine Freundin erzählt:

„Sie hätte gern, dass ihre Mutter mehr Zeit mit ihr verbringt, weil irgendwie, irgendwie, naja, wie soll ich das sagen, irgendwie ist sie schon traurig, weil ihre Mutter immer so lang weg ist und dann fast nie bei ihr ist. Ihre Mutter bringt sie auch nie in die Schule und, naja, da kann man sich schon was dabei denken. Nämlich, dass das Kind arm ist." (Mädchen W 14, Stieffamilie)

Die befragten Eltern aus Kern- und Stieffamilien belegten Kinder in Ein-Eltern-Familien mit Begriffen wie „,arm“, „,benachteiligt“ oder „,entwicklungsgefährdet“. Sie äußerten 
Bewunderung, aber auch Mitleid für alleinerziehende Eltern, deren Situation auf Basis von drei Aspekten als negativ konstruiert wurde: Abwesenheit einer zweiten Elternperson, Zeitdruck sowie Unsicherheit. Erstens wurde die Abwesenheit einer zweiten Elternperson im Alltag als ,Fehlen' interpretiert und als beträchtliches Defizit betrachtet, da eine einzelne erwachsene Person die Verantwortung für das Wohlergehen der Kinder trage und eine Aufteilung von Alltags- und Erziehungsaufgaben nicht möglich sei. Die Argumentation folgte traditionellen Vorstellungen über Geschlechterrollen, wie folgendes Zitat veranschaulicht: „Eine alleinerziehende Mutter muss auch manchmal der Vater sein, der die harte Hand hat. "(Vater W 15, Kernfamilie). Der zweite Aspekt der Benachteiligung war der vermutete höhere Zeitdruck, welcher Ein-Eltern-Familien aufgrund der Notwendigkeit, das Familieneinkommen alleine zu erwirtschaften, kennzeichnen und die Kinder benachteiligen würde. Drittens wurden Ein-Eltern-Familien als instabil und , unsicher" konstruiert, und zwar bezüglich ihrer materiellen Situation, der familialen Alltagsorganisation und des Umgangs mit unvorhergesehenen Herausforderungen. EinEltern-Familien könnten Kindern nicht jene Stabilität und Sicherheit bieten, die ZweiEltern-Familien kennzeichnen würde:

„Es läuft nicht so rund, es ist ein bisschen eckig. Und in dem Moment, wo irgendwas dazwischen kommt, läuft es aus dem Ruder, ja. Weil die sichere Person braucht nur krank sein, ja, dann funktioniert das System nicht mehr, und dann ist es ganz unrund. [...] Also ich glaube, die eine Person muss noch mehr Sicherheit bieten, und auf der lastet noch viel mehr. Also Sicherheit ist, glaube ich, das ganz wichtige Thema. " (Mutter W 08, Kernfamilie).

Wie stellten die Befragten, welche selbst in einer Ein-Eltern-Familie lebten, ihre Situation dar? Die befragten Kinder betrachteten diese familiale Lebensform ebenfalls als nachteilig für Kinder und erachteten Ausmaß, Häufigkeit und Gestaltung der Kontakte zum nicht-residenziellen Elterntei ${ }^{16}$ als besonders relevant. Sie berichteten von negativen Zuschreibungen und diskriminierenden Äußerungen in ihrem Umfeld (durch Bekannte, Schulfreundinnen/Schulfreunde und Pädagog(inn)en) und fühlten sich bei schulischen Anlässen aufgrund der Abwesenheit eines zweiten Elternteils häufig ausgeschlossen. Im Umgang damit beschrieben sie Strategien, welche auf der Betonung bzw. Imitation kernfamilialer Aspekte basierten. Zunächst waren die Kinder bemüht, bei (schulischen) Veranstaltungen bewusst mit beiden leiblichen Elternteilen aufzutreten. War dies nicht möglich, so versuchten jene Kinder, deren alleinerziehende Mutter einen außerhalb lebenden Partner hatte, diesen dafür zu gewinnen. Ein Kind berichtete, dass es diesen im öffentlichen Raum „Papa“ nannte, obwohl er im privaten Kontext mit dem Vornamen angesprochen wurde.

Die befragten AlleinerzieherInnen betonten ebenfalls die negativen Aspekte ihrer Lebensform. Als besondere Herausforderungen betrachteten sie ihre finanzielle Situation, die alleinige Verantwortung sowie die fehlende emotionale und alltagspraktische Entlastung. Sie berichteten von Ausgrenzungen und nahmen eine starke soziale Erwünschtheit von Zwei-Eltern-Familien wahr, in deren Gegenwart ihnen ihr eigener, als defizitär wahrgenommener, Status besonders bewusst wurde. Dabei wurde jeweils Bezug auf die (idealisierte) Kernfamilie genommen, wie die folgenden Zitate exemplarisch zeigen:

16 In Österreich muss bei Vereinbarung der Obsorge beider Elternteile ein hauptsächlicher Aufenthaltsort des Kindes und somit ein Residenz-Elternteil festgelegt werden. 
„Man wird schon ausgegrenzt, das merkt man schon. Man kommt sich immer vor wie das dritte Radl. Sie reden zwar mit einem, aber auch nur das notwendigste. [...] Die sind alle eine intakte Familie, und das haben wir aber nicht. " (Mutter B 25, Alleinerzieherin)

„Prinzipiell würd ich sagen, dass eine Familie, die funktioniert, wo beide Ehepartner da sind und die Kinder, eher Anschluss an eine andere funktionierende Familie findet als eine funktionierende Familie und ein Alleinerzieher mit einem Kind. [...] Ich würde mich nicht so wohl fühlen, gemeinsam mit einer Familie, die funktioniert. " (Vater W 19, Alleinerzieher)

Die befragten alleinerziehenden Eltern nannten zwei Strategien im Umgang mit Stigmatisierung: die Imitation kernfamilialer Strukturen, ähnlich wie von den Kindern beschrieben, sowie die Vermeidung von Kontakten mit Zwei-Eltern-Familien.

\section{Stieffamilien als , modifizierte Kernfamilien ‘}

Vom Großteil der Befragten wurde für Stieffamilien der Begriff „Patchwork-Familie“ verwendet, einige sprachen von ,,gemischten “ oder ,zusammengewachsenen “ Familien. Stieffamilien wurden von den Eltern, die in Kernfamilien leben, zwiespältig betrachtet: einerseits wurden ihnen aufgrund ihrer ,, unklaren “ Familienstruktur, dem vermuteten daraus resultierenden höheren Konfliktpotential sowie den erhöhten Mobilitätsanforderungen Nachteile zugeschrieben. Andererseits wurden Stieffamilien als modifizierte Kernfamilien konstruiert und angenommen, dass diese Nachteile durch das Vorhandensein von zwei Elternpersonen reduziert bzw. kompensiert werden könnten, denn ,, bei Patchwork-Familien ist doch die Familie: Vater, Mutter, Kind. " (Mutter B 30, Kernfamilie). Eltern aus Kernfamilien und Ein-Eltern-Familien leiteten aus der strukturellen Ähnlichkeit mit Kernfamilien Vorteile wie die gemeinsame Übernahme von elterlichen Aufgaben und Verantwortlichkeiten ab. Dies führte zu einer durchaus positiven Einschätzung von Stieffamilen bezüglich ihrer Möglichkeiten, eine förderliche Umgebung für Kinder bereit zu stellen.

Die Sichtweisen der befragten Eltern, welche selbst in Stieffamilien leben, hingen mit dem Komplexitätsgrad ihrer Familien zusammen: Eltern aus einfachen und zusammengesetzten Stieffamilien betonten, dass ihre Familien der gesellschaftlichen Norm der ZweiEltern-Familie entsprächen, und leiteten daraus positive Konsequenzen ab, während Eltern aus komplexen Stieffamilien ihre Familien aufgrund der komplexen Familienkonstellationen als relativ weit entfernt von gesellschaftlichen Konventionen betrachteten ${ }^{17}$. Von Befragten aus allen Stieffamilien-Typen wurde betont, dass sie ihre Familien als „,komplett“ betrachteten, da (mindestens) zwei Elternpositionen im Familienalltag besetzt waren.

Die befragten Kinder in Kernfamilien und Ein-Eltern-Familien schrieben der biologischen Verwandtschaft einen deutlich höheren Stellenwert zu als der Familienstruktur. Sie definierten Stieffamilien in erster Linie als Stiefvaterfamilien, etwa wie folgt: „Da hat man so einen Papa, der eigentlich gar nicht so der Papa ist." (Junge W 12, Kernfamilie). Insgesamt konstruierten die befragten Kinder Stieffamilien deutlich negativer als ihre

17 Diese Typologie basiert auf der Zusammensetzung der im Haushalt lebenden Kinder (Teubner 2002; Feldhaus/Huinink 2011). In einfachen Stieffamilien bringt nur ein Partner Kinder in die Beziehung ein, das Paar hat keine gemeinsamen leiblichen Kinder. In zusammengesetzten Stieffamilien bringen beide Partner Kinder in die Beziehung, es gibt ebenfalls keine gemeinsamen leiblichen Kinder. Komplexe Stieffamilien sind dadurch gekennzeichnet, dass mindestens ein Partner ein Kind einbringt und es zusätzlich mindestens ein gemeinsames Kind gibt. 
Eltern. Sie betonten die ihrer Meinung nach aus der Abwesenheit des „richtigen “ Vaters resultierenden Nachteile und vermuteten aufgrund des Mobilitäts- und Abstimmungsbedarfs eine Überforderung der betroffenen Kinder. Die befragten Kinder in Stieffamilien thematisierten auch Vorteile ihrer Familienform wie z.B. das breitere Spektrum an (stief-) elterlichen Kompetenzen.

Zusammenfassend erwiesen sich für die Konstruktion von Stieffamilien zwei Differenzierungsmerkmale als besonders relevant, nämlich die Dauer des Bestehens (bzw. Alter des Kindes bei Gründung der Stieffamilie) und die Komplexität der Familienstruktur (ableitbar aus der Anzahl an Personen und Verwandtschaftsverhältnissen). Je näher diese beiden Merkmale der Kernfamilie kamen (d.h. längere Dauer, geringere Komplexität), desto eher schrieben die Befragten Stieffamilien positive Auswirkungen auf die kindliche Entwicklung zu.

\section{Zusammenfassung und Diskussion}

Die Beantwortung der Frage, wie Scheidung, Kernfamilien und Nachscheidungsfamilien von den befragten Kindern und Eltern konstruiert werden, lässt sich in drei zentralen Aspekten bündeln:

(1) Scheidung und ihre Folgen werden vielfach dem Desorganisationsmodell entsprechend konstruiert und nicht als familialer Übergang, sondern vielmehr als Auflösung der Familie gesehen. Scheidung und ihre Folgen werden auf der individuellen Ebene verortet und nicht als gesellschaftlich (mit-)bedingt, sondern als privates ,Scheitern' betrachtet. Geschiedenen Eltern wird zugeschrieben, sich zu wenig um den Erhalt der Partnerbeziehung bemüht $\mathrm{zu}$ haben. Kinder geschiedener Eltern werden nicht als Akteurinnen/ Akteure, sondern als passive Opfer einer elterlichen Scheidung konstruiert. Dies lässt u.a. vermuten, dass kindorientierte scheidungsbegleitende Maßnahmen, wie sie in den vergangenen Jahren in Österreich - wenn auch bislang nicht in ausreichendem Maße - ausgebaut wurden, von den Befragten kaum wahrgenommen werden.

(2) Es zeigt sich eine deutliche Hierarchisierung von Lebensformen. Das Bild der Kernfamilie als ,ideale“ Lebensform ist fest in den Köpfen der Befragten verankert und findet durch Zuschreibungen von Überlegenheit in Kompetenz und Moral zusätzliche Festigung. Mit der Konstruktion der Kernfamilie als Ideal und als stabile Umgebung für das Aufwachsen von Kindern ist eine Abwertung davon abweichender Lebensformen verbunden. Ein-Eltern-Familien werden aufgrund der ,fehlenden' Besetzung einer Elternposition als benachteiligt betrachtet und konstruieren sich auch selbst - vor der normativen Folie der Kernfamilie - als defizitäre ,Mangelfamilie“. Der auch in der Forschung lange Zeit verwendete Begriff der „unvollständigen Familie“ (Clason 1989) scheint hier präsent zu sein. Stieffamilien werden aufgrund des alltäglichen Vorhandenseins von zwei Elternpersonen als , komplett" betrachtet, woraus die Zuschreibung umfassender Stabilität resultiert. Dies erinnert an den von Hoffmann-Riem et al. (1994) geprägten Begriff der „Normalisierung als ob“ bzw. den Typus der Stieffamilie als „Quasi-Kernfamilie“, wie er von Wilk (2002) für Österreich dargestellt wurde. 
(3) In der vorliegenden Studie zeigt sich eine weitgehende Ausblendung der innerfamilialen Beziehungen zugunsten einer haushaltszentrierten und familienstrukturell fokussierten Konzeption: Die Verfügbarkeit des zweiten Elternteils wird mit seiner Anwesenheit im Haushalt gleichgesetzt, und die Familienstruktur wird in den Mittelpunkt der Argumentation gestellt. Herausforderungen, welche verschiedene Lebensformen auf der Beziehungsebene an die Familienmitglieder stellen, werden hingegen nur am Rande thematisiert. Daraus resultiert u.a., dass eine elterliche Scheidung mit dem „Verlust“ des nicht-residenziellen Elternteils gleichgesetzt wird. Alternative Modelle der (Ob-)Sorge finden kaum Eingang in die Konstruktionsprozesse und scheinen im sozialen Umfeld der Befragten nicht wahrgenommen bzw. auch nicht gelebt zu werden.

Abschließend werden nun einige Überlegungen bezüglich möglicher Auswirkungen dieser Ergebnisse angestellt. Wird Scheidung als Desorganisation und individuelles „Versagen" konstruiert, so stellt dies gesellschaftliche und politische Bemühungen einer Verbesserung von (rechtlichen, materiellen, organisatorischen) Rahmenbedingungen in Frage. Die Sichtweise von Scheidung als privatem ,Scheitern' impliziert nicht nur eine (weitgehend) private Bewältigung, sondern erhöht auch das Potential einer Stigmatisierung von geschiedenen Personen und ihren Kindern. In Kombination mit normativen Konstruktionen über Scheidung und Vorstellungen, wonach Eltern eine Scheidung zum „Wohle ihrer Kinder“ vermeiden könnten bzw. sollten, kann dies zur Erzeugung eines Klimas der Benachteiligung von geschiedenen Personen und ihren Familien beitragen und Projektionsflächen für eine Instrumentalisierung von Kindern durch die Politik schaffen. Letztlich kann dies zu einer Abnahme des politischen Willlens zur Abmilderung von Scheidungsfolgen und damit zu einer Reduktion von Unterstützungsangeboten beitragen. Das erscheint auch insofern relevant, als die Kernfamilie nach wie vor die Folie bildet, vor welcher staatliche Leistungen und rechtliche Regelungen normiert werden.

Die skizzierte Idealisierung der Kernfamilie erscheint problematisch, weil sie eine sachliche Auseinandersetzung erschweren, die Entstehung von Vorurteilen fördern und so zur Benachteiligung von geschiedenen Personen, Kindern mit geschiedenen Eltern bzw. Personen in nicht-kernfamilialen Lebensformen beitragen kann. Ebenso kann die Ausblendung innerfamilialer Beziehungen zugunsten haushaltszentrierter und familienstruktureller Konzeptionen das Verständnis für die Herausforderungen, welche familiales Zusammenleben in unterschiedlichen Konstellationen mit sich bringt, verringern. Dies kann zu verkürzten, undifferenzierten Sichtweisen über Familien beitragen.

Aus diesen möglichen Auswirkungen resultieren Herausforderungen für Sozialpolitik und Familienwissenschaft. Folgen sozialpolitische Maßnahmen einem konservativen Ansatz, so könnten sie darauf abzielen, Kernfamilien aufgrund ihrer hohen normativen Akzep$\operatorname{tanz}$ zu stärken und eine Abwertung davon abweichender Lebensformen in Kauf zu nehmen. Progressive Ansätze würden eher darauf fokussieren, Kindern und Eltern unabhängig von der Familienform ein adäquates Lebensumfeld zu bieten und entsprechende Rahmenbedingungen dafür zu schaffen. Die Bereitstellung ausreichender Mittel für Unterstützungsmöglichkeiten, sowohl im Vorfeld von Scheidungen als auch danach, erscheint dafür unabdingbar (z.B. Mediation, Kinderbegleitung, umfassende Beratungs- und Therapieangebote).

Schließlich ist angesichts zählebiger Ideale und geringen Wissensstands die Familienwissenschaft gefordert, ihre Ergebnisse in angemessener Weise zu disseminieren und diese den vorhandenen, auch medial transportierten, Bildern, Begriffen und Etikettierun- 
gen entgegen zu stellen. Insbesondere wäre das Bewusstsein dafür zu stärken, dass die Gestaltungs- und Entwicklungsmöglichkeiten unterschiedlicher Lebensformen auch von den jeweiligen gesellschaftlichen Rahmenbedingungen abhängig sind. Eine verstärkte, zielgerichtete Verbreitung wissenschaftlicher Befunde könnte damit zum Abbau bestehender Mythen und Vorurteile beitragen.

\section{Literatur}

Adams, M. \& Coltrane, S. (2007). Framing divorce reform: Media, morality, and the politics of family. Family Process, 46, 1, S. 17-34.

Alt, C. \& Lange, A. (2004). Deine Familie, meine Familie? Die Perspektiven von Müttern und ihren Kindern. Zeitschrift für Familienforschung, 16, 2, S: 111-129.

Alt, C. \& Lange, A. (2011). Kindschaftskonstellationen in Vater-Mutter-Familien. In: Schwab, B. \& Vaskovics, L. A. (Hrsg.), Pluralisierung von Elternschaft und Kindschaft. Familienrecht, -soziologie und -psychologie im Dialog. Opladen, Famington Hills, MI: Verlag Barbara Budrich (Sonderheft 8 der Zeitschrift für Familienforschung/Journal of Family Research), S. 139-156.

Amato, P. R. (2000). The consequences of divorce for adults and children. Journal of Marriage and Family, 62, 4, S.1269-1287.

Anyan, S. \& Pryor, J. (2002). What is in a family? Adolescent perceptions. Children \& Society, 16, S. 112.

Berger, P. L. \& Kellner, H. (1965). Die Ehe und die Konstruktion der Wirklichkeit. Soziale Welt, 16, 3, S. 220-235.

Berger, P. L. \& Luckmann, T. (1969). Die gesellschaftliche Konstruktion der Wirklichkeit. Eine Theorie der Wissenssoziologie. Frankfurt am Main: Fischer.

Birney, D. P. \& Sternberg, R. J. (2011). The development of cognitive abilities. In: Bornstein, M. H. \& Lamb, M. E. (Hrsg.), Developmental science. An advanced textbook. New York: Psychology Press, S. 353-388.

Capello, M. (2005). Photo interviews. Eliciting data through conversations with children. Field Methods, 17, 2, S. 170-182.

Castrén, A.-M. (2008). Post-divorce family configurations. In: Widmer, É. D. \& Jallinoja, R. (Hrsg.), Beyond the nuclear family: Families in a configurational perspective. Bern: Peter Lang, S. 233253.

Clark-Ibáñez, M. (2004). Framing the social world through photo-elicitation interviews. American Behavioral Scientist, 47, 12, S. 1507-1527.

Clason, C. (1989). Die Ein-Eltern-Familie oder die Ein-Elter-Familie. In: Nave-Herz, R. \& Markefka, M. (Hrsg.), Handbuch der Familien- und Jugendforschung. Band 1: Familienforschung. Neuwied, Frankfurt: Luchterhand, S. 413-422.

Coltrane, S. \& Adams, M. (2003). The social construction of the divorce 'problem': Morality, child victims, and the politics of gender. Family Relations, 52, 4, S. 363-372.

Cook, T. \& Hess, E. (2007). What the camera sees and from whose perspective. Fun methodologies for engaging children in enlightening adults. Childhood, 14, 1, S. 29-45.

Cowan, P. A. \& Hetherington, E. M. (1991). Family transitions. Advances in family research. Hillsdale: Erlbaum.

Croghan, R., Griffin, C., Hunter, J. \& Phoenix, A. (2008). Young people's constructions of self: Notes on the use and analysis of the photo-elicitation methods. International Journal of Social Research Methodology, 11, 4, S. 345-356.

Cyprian, G. (2003). Familienbilder als Forschungsthema. In: Cyprian, G. \& Heimbach-Steins, M. (Hrsg.), Familienbilder. Interdisziplinäre Sondierungen. Opladen: Leske + Budrich, S. 9-19.

Dekovic, M. \& Buist, K. L. (2005). Multiple perspectives within the family. Journal of Family Issues, 26,4 , S. 467-490. 
Denham, S., Warren, H., Salisch, M. v., Benga, O., Chin, J.-C. \& Geangu, E. (2011). Emotions and social development in childhood. In: Smith. P. K, \& Hart, C. H. (Hrsg.), The Wiley-Blackwell handbook of childhood social development. Chichester: Wiley-Blackwell, S. 413-433.

Diefenbach, H. \& Opp, K.-D. (2007). When and why do people think there should be a divorce? Rationality and Society, 19, 4, S. 485-517.

Feldhaus, M. \& Huinink, J. (2011). Multiple Elternschaften in Deutschland - eine Analyse von Elternschaft in Folgepartnerschaften. In: Schwab, D. \& Vaskovics, L. A. (Hrsg.), Pluralisierung von Elternschaft und Kindschaft. Familienrecht, -soziologie und -psychologie im Dialog. Opladen, Farmington Hills, MI: Verlag Barbara Budrich (Sonderheft der Zeitschrift für Familienforschung/ Journal of Family Research), S. 77-104.

Flowerdew, J. \& Neale, B. (2003). Trying to stay apace: Children with multiple challenges in their postdivorce family lives. Childhood, 10, 2, S: 147-162.

Froschauer, U. \& Lueger, M. (2003). Das qualitative Interview. Wien: WUV.

Fthenakis, W. (1995). Ehescheidung als Übergangsphase (Transition) im Familienentwicklungsprozess. In: Perrez, M., Lambert, J,-L., Ermert, C. \& Plancherel, B. (Hrsg.), Familie im Wandel. Bern: Huber (Freiburger Beiträge zur Familienforschung), S.63-95.

Geulen, D. (2010). Sozialisationstheoretische Ansätze. In: Krüger, H.-H. \& Grunert, C. (Hrsg,), Handbuch Kindheits- und Jugendforschung. Wiesbaden: VS Verlag für Sozialwissenschaften, S. 85-102.

Glaser, B. \& Strauss, A. (1998). Grounded Theory. Strategien qualitativer Forschung. Bern: Huber.

Halman, L., Luijkx, R. \& Zundert, M. van (2005). The atlas of European values. Leiden: Brill.

Hamachers-Zuba, U., Lehner, E. \& Tschipan, C. (2009). Partnerschaft, Familie und Geschlechterverhältnisse in Österreich. In: Friesl, C., U. Hamachers-Zuba, U. \& Polak, R. (Hrsg.) Die ÖsterreicherInnen. Wertewandel 1990-2008. Wien: Czernin, S. 87-142.

Harden, J., Backett-Milburn, K., Hill, M. \& MacLean, A. (2010). Oh, what a tangled web we weave: Experiences of doing 'multiple perspectives' research in families. International Journal of Social Research Methodology, 13, 5, S. 441-452.

Herzer, M. (1998). Ehescheidung als sozialer Prozess. Opladen: Westdeutscher Verlag.

Hill, P. B. \& Kopp, J. (1999). Ehescheidung: Historische Entwicklungen und theoretische Erklärungen. In: Klein, T. \& Kopp, J. (Hrsg.), Scheidungsursachen aus soziologischer Sicht. Würzburg: Ergon, S. 23-42.

Hoffmann-Riem, C., Hoffmann-Riem, W., Pieper, M. \& Riemann, G. (1994). Elementare Phänomene der Lebenssituation: Ausschnitte aus einem Jahrzehnt soziologischen Arbeitens. Weinheim: Deutscher Studienverlag.

Hölscher, B. (2008). Sozialisation, Sozialisationskontexte, schichtspezifische Sozialisation. In: Willems, H. (Hrsg.), Lehr(er)buch Soziologie. Für die pädagogischen und soziologischen Studiengänge. Band 2. Wiesbaden: Verlag für Sozialwissenschaften, S. 747-772.

Hurrelmann, K. (2002). Einführung in die Sozialisationstheorie. Weinheim, Basel: Beltz.

Kalmijn, M. \& Uunk, W. (2007). Regional value differences in Europe and the social consequences of divorce: A test of the stigmatization hypothesis. Social Science Research, 36, 447-468.

Kohlberg, L. (1974). Zur kognitiven Entwicklung des Kindes. Frankfurt am Main: Suhrkamp.

Lange, A. \& Kränzl-Nagl, R. (2010). Familie im Spiegel öffentlicher und privater Diskurse - Bausteine für familienrhetorische Betrachtungen. In: Bundesministerium für Wirtschaft, Familie und Jugend (Hrsg.),, 5. Familienbericht 1999-2009. Die Familie an der Wende zum 21. Jahrhundert. Band 1. Wien: Bundesministerium für Wirtschaft, Familie und Jugend, S: 91-125.

Lüscher, K. (1997). Familienrhetorik, Familienwirklichkeit und Familienforschung. In: Vaskovics, L. A. (Hrsg.), Familienleitbilder und Familienrealitäten. Opladen: Leske + Budrich, S. 50-69.

Lüscher, K., Wehrspaun, M. \& Lange, A. (1989). Familienrhetorik - über die Schwierigkeit, „Familie“ zu definieren. Zeitschrift für Familienforschung, 2, 1, S. 61-76.

Miles, N. J. \& Servaty-Seib, H. L. (2010). Parental marital status and young adult offspring's attitudes about marriage and divorce. Journal of Divorce and Remarriage, 51, 4, S. 209-220.

Miller, P. H. (2011). Piaget's theory. Past, present, and future. In: Goswami, U. (Hrsg.), The WileyBlackwell Handbook of childhood cognitive development. Chichester: Wiley-Blackwell, S. 649672. 
Moon, M. (2011). The effects of divorce on children: Married and divorced partners' perspectives. Journal of Divorce and Remarriage, 52, 5, S. 344-349.

Moxnes, K. (2003). Risk factors in divorce: Perceptions by the children involved. Childhood, 10, 2, S. 131-146.

Nave-Herz, R. (2006). Ehe- und Familiensoziologie. Eine Einführung in Geschichte, theoretische Ansätze und empirische Befunde. München: Juventa.

Nelson, M. K. (2006). Single mothers „do“ family. Journal of Marriage and Family, 68, 4, S. 781-795.

OECD (2010). Familiy Database. http://www.oecd.org/els/social/family/database [Stand: 2011-03-10].

Oerter, R. \& Montada, L. (2008) (Hrsg.). Entwicklungspsychologie. Weinheim, Basel: Beltz.

Oswald, R. F., Blume, L. B. \& Marks, S. R. (2005). Decentering heteronormativity. A model for family studies. In: Bengtson, V. L., Acock A. C., Allen, K. R., Dilworth-Anderson, P. \& Klein, D. M. (Hrsg.), Sourcebook of family theory and research Thousand Oaks, London, New Delhi: Sage, S. 143-154.

Piaget, J. (1972). The psychology of the child. New York: Basic Books.

Piaget, J. (1986). Das moralische Urteil beim Kinde. München: Klett-Cotta.

Ribbens McCarthy, J., Holland, J. \& Gillies, V. (2003). Multiple perspectives on the 'family' lives of young people: Methodological and theoretical issues in case study research. International Journal of Social Research Methodology, 6, 1, S. 1-23.

Rigg, A. \& Pryor, J. (2007). Children's perceptions of families: What do they really think? Children and Society, 21, S. 17-23.

Robinson, M., Butler, I., Scanlan, L., Douglas, G. \& Murch, M. (2003). Children's experience of their parents' divorce. In: Jensen, A.-M. \& McKee, L. (Hrsg.), Children and the changing family. Between transformation and negotiation. London, New York: Routledge/Falmer, S. 76-89.

Roe, A., Bridges, L., Dunn, J. \& O'Connor, T. (2006). Young children's representations of their families: A longitudinal follow-up study of family drawings by children living in different family settings. International Journal of Behavioral Development, 30, 6, S. 529-536.

Röhr-Sendlmeier, U. M. \& Greubel, S. (2004). Die Alltagssituation von Kindern in Stieffamilien und Kernfamilien im Vergleich. Zeitschrift für Familienforschung, 16, 1, S. 56-71.

Sander, E., Endepohls-Ulpe, M. \& Gollia, A. (2005). Scheidungskinder im Urteil von Lehrerinnen und Lehrern. Psychologie in Erziehung und Unterricht, 52, S. 272-280.

Schmitz, H. \& Schmidt-Denter, U. (1999). Die Nachscheidungsfamilie sechs Jahre nach der elterlichen Trennung. Zeitschrift für Familienforschung, 11, 3, S. 28-55.

Schneewind, K. A. \& Walper, S. (2008). Kinder in verschiedenen Familienformen. In: Hasselhorn, M. \& Silbereisen, R. K. (Hrsg.) Entwicklungspsychologie des Säuglings- und Kindesalters. Göttingen: Hogrefe, S. 571-616.

Schneider, N. F. (2008). Grundlagen der sozialwissenschaftlichen Familienforschung - Einführende Betrachtungen. In: Schneider, N. F. (Hrsg.), Lehrbuch Moderne Familiensoziologie. Opladen, Farmington Hills, MI: Verlag Barbara Budrich, S. 9-40.

Smart, C., Neale, B. \& Wade, A. (2001). The changing experience of childhood. families and divorce. Cambridge: Polity Press.

Smith, A. B., Taylor, N. J. \& Tapp, P. (2003). Rethinking children's involvement indecision-making after parental separation. Childhood, 10, 2, S. 201-216.

Smith, D. E. (1993). The standard North American family: SNAF as an ideological code. Journal of Family Issues, 14, 1, S. 50-65.

Statistik Austria (2010a). Ehescheidungen. http://www.statistik.at/web_de/presse/049433 [Stand: 201102-21].

Statistik Austria (2010b). Familien- und Haushaltsstatistik 2009. Ergebnisse der Mikrozensus-Arbeitskräfteerhebung. Wien: Verlag Österreich.

Statistik Austria (2011a). Ehescheidungen. http://www.statistik.at/web_de/dynamic/presse/056777 [Stand: 2011-06-21].

Statistik Austria (2011b). Familien- und Haushaltsstatistik 2010. Wien: Verlag Österreich.

Statistik Austria (2011c). Statistisches Jahrbuch 2011. Wien: Verlag Österreich. 
Strauss, A. \& Corbin, J. (1990). Basics of qualitative research: Grounded theory procedures and techniques. Newbury Park, London, New Delhi: Sage.

Teubner, M. (2002). Stieffamilientypen und haushaltsübergreifende Stiefkonstellationen. In: Bien, W., Hartl, A. \& Teubner, M. (Hrsg.), Stieffamilien in Deutschland. Eltern und Kinder zwischen Normalität und Konflikt. Opladen: Leske + Budrich, S. 51-82.

Théry, I. (1988). Die Familien nach der Scheidung: Vorstellungen, Normen, Regulierungen. In: Lüscher, K., Schultheis, F. \& Wehrspaun, M. (Hrsg.), Die postmoderne Familie. Konstanz: Universitätsverlag, S. 84-97.

Toppe, S. (2009). Rabenmütter, Supermuttis, abwesende Väter? Familien(leit)bilder und Geschlechtertypisierungen im Kinderarmutsdiskurs in Deutschland. In: Villa P. \& Thiessen, B. (Hrsg.), Mütter Väter: Diskurse, Medien, Praxen. Münster: Westfälisches Dampfboot, S. 107-127.

Toth, K. \& Kemmelmeier, M. (2009). Divorce attitudes around the world. Distinguishing the impact of culture on evaluations and attitude structure. Cross-Cultural Research, 43, 3, S. 280-297.

Usdansky, M. L. (2009). A weak embrace: Popular and scholarly depictions of single-parent families, 1900-1998. Journal of Marriage and Family, 71, S. 209-225.

Watson, M. W. \& Amgott-Kwan, T. (1984). Development of family-role concepts in school-age children. Developmental Psychology, 20, S. 953-959.

Wendt, E.-V. \& Walper, S. (2007). Entwicklungsverläufe von Kindern in Ein-Eltern- und Stieffamilien. In: Alt, C. (Hrsg.), Kinderleben - Start in die Grundschule. Band 3: Ergebnisse aus der zweiten Welle. Wiesbaden: VS Verlag für Sozialwissenschaften, S. 211-242.

Wernhart, G. \& Neuwirth, N. (2007). Geschlechterrollenwandel und Familienwerte (1988-2002). Österreich im europäischen Vergleich. Ergebnisse auf Basis des ISSP 1988, 2002. Wien: Österreichisches Institut für Familienforschung (ÖIF Working Paper 54).

Wilk, L. (2002). Stieffamilien in Österreich. In: Bien, W., Hartl, A. \& Teubner, M. (Hrsg.), Stieffamilien in Deutschland. Eltern und Kinder zwischen Normalität und Konflikt. Opladen: Leske + Budrich, S. 245-284.

Witzel, A. (2000). Das problemzentrierte Interview. Forum Qualitative Sozialforschung, 1, 1, Art. 22, http://nbn-resolving.de/urn:nbn:de:0114-fqs0001228.

Zartler U., Marhali A., Starkbaum, J. \& Richter, R. (2009). Familien in Nahaufnahme. Eltern und ihre Kinder im städtischen und ländlichen Raum. Wien: Bundesminsterium für Wirtschaft, Familie und Jugend.

Zartler, U. (2010). Multiple perspectives in qualitative family research: crafting and conducting research projects. Family Science, 1, 3-4, S. 173-182.

Zartler, U. (2011). Reassembling families after divorce. In: Jallinoja, R. \& Widmer, E. D. (Hrsg.), Families and kinship in contemporary Europe: Rules and practices of relatedness. Basingstoke: Palgrave Macmillan, S. 178-191.

Eingereicht am/Submitted on: 04.04.2011

Angenommen am/Accepted on: 06.09.2011

Anschrift der Autorin/Address of the author:

\section{Dr. Ulrike Zartler}

Universität Wien

Institut für Soziologie

Rooseveltplatz 2

1090 Wien

Österreich/Austria

E-Mail: ulrike.zartler@univie.ac.at 